\title{
Linear Instability Analysis Method with Three-Dimensional Finite Element Model of Lean Premixed Combustor*
}

\author{
Kazufumi IKEDA**, Keisuke MATSUYAMA**, Mitsunori ISONO** \\ and Masaharu NISHIMURA*** \\ ** Takasago Research \& Development Center, Mitsubishi Heavy Industries, Itd., \\ 2-1-1 Shinhama Arai-cho, Takasago, Hyogo 676-8686 JAPAN \\ E-mail: kazufumi_ikeda@mhi.co.jp \\ *** Department of Mechanical Engineering, Tottori University, \\ 4-101 Koyama-cho South, Tottori, Tottori Pref., 680-8552 JAPAN \\ E-mail: mnishimura@mech.tottori-u.ac.jp
}

\begin{abstract}
Combustion oscillation of a self-excited combustion-acoustic phenomenon occurs inside the gas turbine combustor. In general, excessive pressure fluctuation of combustion oscillation may impair the gas turbine engine operation and may result in hardware damages. Hence, combustion oscillation has been one of the problems of the gas turbine development. For predicting the combustion-acoustic instability on the designing stage, we have developed one-dimensional linear instability analysis method and verified the analysis accuracy by premixed combustor laboratory tests. According to the prior efforts, the basic framework of combustionacoustic system has been captured, and then, we have known that not only precise analysis method but also high precise model parameters are needed for accurate analysis. So in this paper, to achieve this, three-dimensional combustion-acoustic analysis method with finite element model which can simulate precisely the geometry and combustion state of an actual gas turbine combustor is developed. Further, for the calculation efficiency, the approximation calculation method for evaluating the complex eigenvalues of finite element model is proposed and its accuracy is validated at simple examples.
\end{abstract}

Key words: Gas Turbine, Combustor, Combustion Oscillation, Acoustics, Analysis

\section{Introduction}

Combustion oscillation of a self-excited combustion-acoustic phenomenon occurs inside the gas turbine combustor in operating at higher temperatures with reducing NOx and other gaseous emissions. In general, excessive pressure fluctuation of combustion oscillation may impair the gas turbine engine operation and may result in hardware damages. Hence, combustion oscillation has been one of the problems of the gas turbine development [1]-[3]. The mechanism of combustion-acoustic instability is well researched, then many analytical or experimental researches have been discussed [4]-[9]. According to Rayleigh [4] referenced by many researchers, the combustion-acoustic instability criterion is described as the following inequality

$$
\int_{t_{0}} \int_{V} p^{\prime} q^{\prime} \mathrm{d} V \mathrm{~d} t>\int_{t_{0}} \int_{V} \Phi \mathrm{d} V \mathrm{~d} t
$$


where $p^{\prime}, q^{\prime}, V, \Phi, t$ and $t_{0}$ are perturbations of pressure and heat release density, combustor volume, acoustic energy dissipation, time, and period of oscillation, respectively. The left hand side of Rayleigh's criterion (1) means amplification rate of dynamic variables $p^{\prime}$ and $q^{\prime}$ which are correlated with each other, and the right hand side means damping rate of them. Combustion-acoustic instability occurs when Rayleigh's criterion (1) is satisfied. Damping rate increase of the right hand side of Rayleigh's criterion (1) can be considered an instability suppression method. The design by this suppression method is relatively easy, so that the detailed combustion predictions are not needed. Focusing on this point, we have developed the robust gas turbine combustor with acoustic liner [2] as shown in Figure 1.

However, in particular at low frequencies, there is a problem that the dimension of the suppression device is to be large. So, we have focused on amplification rate decrease of the left hand side of Rayleigh's criterion (1) at low frequencies due to tuning the interaction between the combustion dynamics and the acoustic dynamics, and also to achieve this, one-dimensional combustion-acoustic linear instability analysis method [3] has been developed and verified at the simple combustor model as shown in Figure 2. According to this prior paper, the basic framework of combustion-acoustic system has been captured, and then, we have known that not only precise analysis method but also high precise model parameters are needed for accurate combustion-acoustic instability analysis. To achieve this, three-dimensional combustion-acoustic analysis method which can simulate precisely the geometry and combustion state of an actual gas turbine combustor is needed.

So, in this paper, we extend one-dimensional analysis concept of the prior paper [3] to three-dimensional generic analysis method by using finite element method. At the next section, three-dimensional differential equations of combustion-acoustic system are formulated. These equations are reformulated as finite element models in Section 3. The instability analysis method is discussed in Section 4. Section 5 and 6 shows the validation results at simple examples. Conclusions are summarized in the final section.

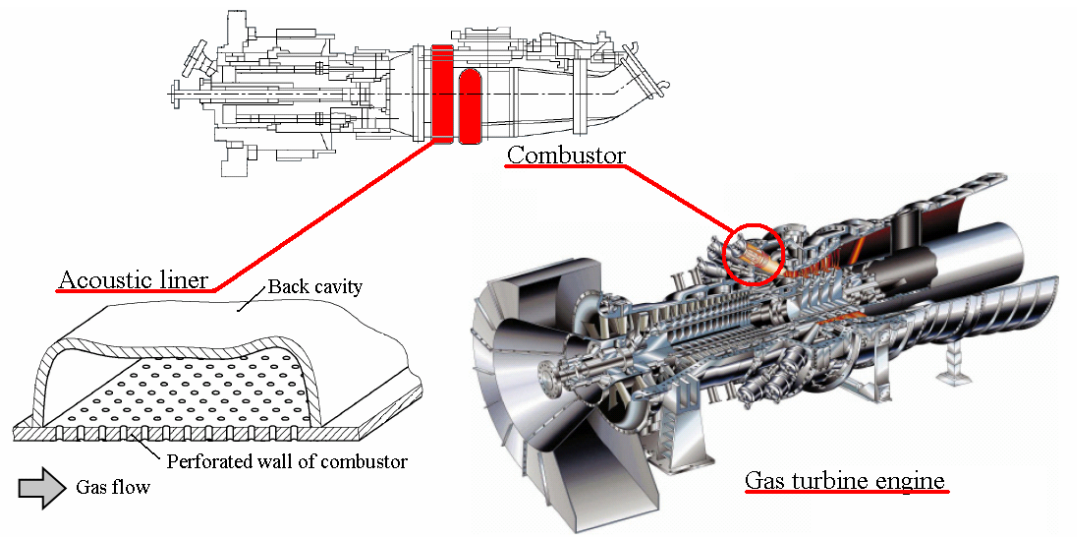

Fig. 1 Robust gas turbine combustor with acoustic liner [2].

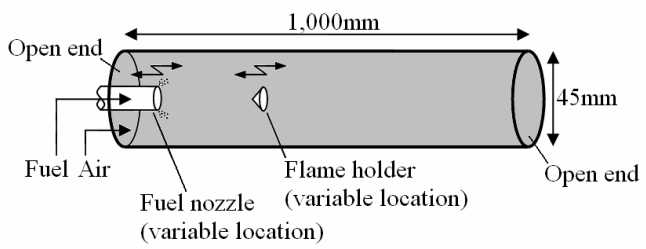

Fig. 2 One-dimensional model of linear instability analysis of lean premixed combustion [3]. 


\section{Formulations of basic equations of combustion-acoustic system}

In this section, basic differential equations of combustion-acoustic system are formulated. Basic equations consist of acoustic system driven by heat release fluctuation, heat release fluctuation driven by acoustic system, and boundary conditions.

\subsection{Acoustic system driven by heat release fluctuation}

Basic equations of the fluid dynamics can be described as

$$
\begin{aligned}
& \frac{D \rho}{D t}+\rho \nabla \cdot \mathbf{u}=0, \\
& \rho \frac{D \mathbf{u}}{D t}=-\nabla p+\Psi, \\
& \rho T \frac{D S}{D t}=q, \\
& \mathrm{~d} p-c^{2} \mathrm{~d} \rho=(\gamma-1) \rho T \mathrm{~d} S, \\
& p=\rho R T,
\end{aligned}
$$

where $\rho, p, \mathbf{u}, q, S, T, \gamma, R, c$ and $D / D t(\equiv \partial / \partial t+\mathbf{u} \cdot \nabla)$ are density, pressure, velocity, heat release density, entropy, temperature, ratio of specific heats, gas constant, speed of sound, Lagrange differential, respectively. Also, $\Psi$ represents viscosity and external force terms. The nonlinear wave equation equivalent to basic equations (2)-(6) can be derived [3], namely

$$
\begin{aligned}
\frac{\partial^{2} p}{\partial t^{2}}-c^{2} \nabla^{2} p= & (\gamma-1) \frac{D q}{D t}-(\gamma-1) \rho T \nabla S \cdot \frac{D \mathbf{u}}{D t}+\frac{D c^{2}}{D t} \frac{D \rho}{D t} \\
& -2 \mathbf{u} \cdot \nabla \frac{\partial p}{\partial t}-\mathbf{u} \cdot(\mathbf{u} \cdot \nabla) \nabla p \\
& +2 \rho c^{2}(\nabla \cdot \mathbf{u})^{2}+\rho c^{2} \nabla \cdot[(\mathbf{u} \cdot \nabla) \mathbf{u}-\mathbf{u}(\nabla \cdot \mathbf{u})] \\
& -c^{2} \nabla \cdot \Psi .
\end{aligned}
$$

When nonlinear terms, viscosity and external force terms, and advection terms which are written like as $(\overline{\mathbf{u}} \cdot \nabla) X^{\prime}$ with respect to a perturbation value $X^{\prime}$ can be neglected, the acoustic equation driven by heat release fluctuation can be obtained as

$$
\nabla^{2} \hat{p}^{\prime}+k^{2} \hat{p}^{\prime}=-i \omega \frac{\gamma-1}{c^{2}} \hat{q}^{\prime}+i \omega \frac{\gamma-1}{c^{2}} \bar{\rho} \bar{T} \nabla \bar{S} \cdot \hat{\mathbf{u}}^{\prime}
$$

in Fourier domain, where $\mathbf{\bullet}, \bullet^{\prime}, \hat{\bullet}, i, k \equiv \omega / c, \omega \equiv 2 \pi f$, and $f$ are the mean value, perturbation value, variables in Fourier domain, the imaginary unit, wave number, angular frequency, and frequency, respectively. A further approximation of the equation (8) with assuming isobaric process $(\gamma-1) \bar{\rho} \bar{T} \nabla \bar{S} \cong-c^{2} \nabla \bar{\rho}$ is consistent with the acoustic equation discussed by Dowling [5] and Hedge et al. [6], that is,

$$
\omega^{2} \hat{p}^{\prime}+\bar{\rho} c^{2} \nabla \cdot\left(\frac{\nabla \hat{p}^{\prime}}{\bar{\rho}}\right)=-i \omega(\gamma-1) \hat{q}^{\prime}
$$




\subsection{Heat release fluctuation driven by acoustic system}

In this section, with assumed that the mean of combustion has been already obtained by numerical simulation, consider the perturbation of combustion having a coherency with the acoustic resonance [3]. Referenced Williams [7], consider heat release sources which consist of combustion reaction $q_{C}$, heat conduction $q_{W}$, and mixing diffusion $q_{D}$ as of,

$$
\begin{aligned}
& \rho T\left(\frac{\partial S}{\partial t}+\mathbf{u} \cdot \nabla S\right)=q, \quad q=q_{C}+q_{W}+q_{D}, \\
& q_{C} \equiv \sum_{b} h_{b} w_{b}, \quad q_{W} \equiv \nabla \cdot \mathbf{q}, \quad q_{D} \equiv \nabla \cdot \sum_{b} \rho h_{b} Y_{b} \mathbf{v}_{b},
\end{aligned}
$$

where $h_{b}, w_{b}, Y_{b}, \mathbf{v}_{b}$, and $\mathbf{q}$ are enthalpy of chemical element $b$, fuel consumption rate by reaction, concentration, mixing diffusion rate, heat flux, respectively. Variables without subscript $b$ mean the average value of chemical species.

In this paper, the mechanism due to combustion reaction is assumed dominant, namely $q \cong q_{C}$. Consider the comprehensive reaction model which is assumed to consist of comprehensive species, namely fuel, air, and combusted mixture gases. Thus, define variables by the average over chemical species, e.g. fuel/air ratio $Y$ means the mass concentration of fuel. According to the basic theory (11), heat release fluctuation $q^{\prime} \cong q_{C}^{\prime}$ would depend on enthalpy fluctuation and reaction rate fluctuation $w^{\prime}$. Of these, as a mechanism that may have an acoustical coherence, this paper focuses on the latter. Further, assume lean premixed combustion. According to the chemical kinetics theory, reaction rate would depend on molarities of reactants. Approximated this, this paper considers the reaction rate model as of $w \propto Y^{N}$. Namely, consider reaction rate fluctuation $w^{\prime}$ caused by fuel/air ratio fluctuation $Y^{\prime}$. And then, assume the quasi-static relations among these variables as $q^{\prime}=(\bar{q} / \bar{w}) w^{\prime}, w^{\prime}=N(\bar{w} / \bar{Y}) Y^{\prime}$ and $N \equiv(\bar{Y} / \bar{w}) \partial w / \partial Y$.

Fuel/air ratio fluctuation is generated by fuel/air supply fluctuation at the fuel nozzle position, and flows to the combustion region by advection, and is consumed by combustion reaction. This fuel mass balance mechanism can be described as

$$
\frac{\partial}{\partial t}(\rho Y)+\nabla \cdot(\rho \mathbf{u} Y)=m \delta\left(\mathbf{x}-\mathbf{x}_{N}\right)-w
$$

where $\mathbf{x}_{N}$ and $m\left(\equiv \rho_{N} u_{N} A_{N}\right)$ are the fuel nozzle position and fuel mass injection rate, and where, $\rho_{N}, u_{N}$, and $A_{N}$ are fuel density, fuel injection velocity, and cross-sectional area of the nozzle hole, respectively. The function $\delta(\cdot)$ is Dirac's delta function. For the simplicity, concentration mixing diffusion effect has been neglected at this equation. Circulation flow fluctuation or, chemical mechanism itself is not vibration mechanism essentially. So, it would be difficult having a consistency with the acoustic resonance, with the exception of rare cases. Consider a stream tube which is consist of stream lines of the mean flow, and consider the curvilinear coordinates along the mean flow vector $\overline{\mathbf{u}}$. In absence of diffusion, fuel mass is conserved along a stream tube. By averaging the equation (12) and the equation of continuity (2) over cross-section of a stream tube, the conservation equations can be written in one-dimensional along the curvilinear coordinates in Fourier domain,

$$
\begin{aligned}
& i \frac{\omega}{\bar{v}} \hat{Y}^{\prime}+\frac{\partial \hat{Y}^{\prime}}{\partial \xi_{u}}=\frac{\bar{m}}{\bar{\rho} \bar{v} A_{u}} \hat{\varphi}^{\prime} \delta\left(\xi_{u}-\xi_{u, N}\right)-\frac{\bar{w}}{\bar{\rho} \bar{v}}\left(\frac{\hat{w}^{\prime}}{\bar{w}}-\frac{\hat{v}^{\prime}}{\bar{v}}\right), \\
& \frac{\partial \bar{Y}}{\partial \xi_{u}}=\frac{\bar{m}}{\bar{\rho} \bar{v} A_{u}} \delta\left(\xi_{u}-\xi_{u, N}\right)-\frac{\bar{w}}{\bar{\rho} \bar{v}}, \quad \frac{\partial}{\partial \xi_{u}}\left(\bar{\rho} \bar{v} A_{u}\right)=0,
\end{aligned}
$$




$$
\varphi^{\prime} \equiv\left(\frac{m^{\prime}}{\bar{m}}-\frac{v^{\prime}}{\bar{v}}\right)_{\xi_{u}=\xi_{u, N}}, \quad \bar{v} \equiv|\overline{\mathbf{u}}|, \quad \frac{v^{\prime}}{\bar{v}} \equiv \frac{\overline{\mathbf{u}} \cdot \mathbf{u}^{\prime}}{|\overline{\mathbf{u}}|^{2}}
$$

where $\xi_{u}, \xi_{u, N}, v, A_{u}$, and $\varphi^{\prime}$ are the curvilinear coordinates along the mean flow vector $\overline{\mathbf{u}}$, the fuel nozzle position on the coordinates of $\xi_{u}$, a representation of velocity $\mathbf{u}$ on the coordinates of $\xi_{u}$, cross-section of a stream tube of a spatial function defined on the coordinates of $\xi_{u}$, and the normalized fuel/air ratio fluctuation at the fuel nozzle position, respectively. Here, by assuming a longer acoustic wavelength than the scale of advection, the acoustical disturbance propagating between adjacent stream tubes is neglected. At the upstream region of flame, the second terms of the right hand side of the equations (13) and (14) can be deleted, so fuel/air ratio fluctuation can be solved as

$$
\bar{Y}=\frac{\bar{m}}{\bar{\rho} \bar{v} A_{u}} h\left(\xi_{u}-\xi_{u, N}\right), \quad \hat{Y}^{\prime}=\lambda \bar{Y} \hat{\varphi}^{\prime} \exp \left[-i \omega \int_{\xi_{u, N}}^{\xi_{u}} \frac{1}{\bar{v}} \mathrm{~d} \xi_{u}\right]
$$

where $h(\cdot)$ means Heaviside's step function. In actual practice, the fluctuation advection above would be influenced by hydrodynamic disturbance, and thus the fluctuation would diffuse. So, the variable $\lambda$ has been introduced for correcting this diffusion effect which has been neglected in the equation (12). At the combustion region, fuel consumption with reaction should be considered, namely the second terms of the right hand side of the equations (13) and (14). By integrating these equations over an interval including the flame position $\xi_{u}=\xi_{u, F}$, the fuel mass balance at the combustion region can be written as

$$
\begin{aligned}
& \int_{\xi_{u, F}-\varepsilon}^{\xi_{u, F}+\varepsilon} \hat{w}^{\prime} A_{u} \mathrm{~d} \xi_{u}+i \omega \int_{\xi_{u, F}-\varepsilon}^{\xi_{u, F}+\varepsilon} \bar{\rho} \hat{Y}^{\prime} A_{u} \mathrm{~d} \xi_{u} \\
& \quad=\left.\bar{\rho} \bar{v} \hat{Y}^{\prime} A_{u}\right|_{\xi_{u}=\xi_{u, F}-\varepsilon}-\left.\bar{\rho} \bar{v} \hat{Y}^{\prime} A_{u}\right|_{\xi_{u}=\xi_{u, F}+\varepsilon}+\int_{\xi_{u, F}-\varepsilon}^{\xi_{u, F}+\varepsilon} \bar{w} \bar{v} \hat{v}^{\prime} A_{u} \mathrm{~d} \xi_{u}, \\
& \int_{\xi_{u, F}-\varepsilon}^{\xi_{u, F}+\varepsilon} \bar{w} A_{u} \mathrm{~d} \xi_{u}=\left.\bar{\rho} \bar{v} \bar{Y} A_{u}\right|_{\xi_{u}=\xi_{u, F}-\varepsilon}-\left.\bar{\rho} \bar{v} \bar{Y} A_{u}\right|_{\xi_{u}=\xi_{u, F}+\varepsilon} .
\end{aligned}
$$

Each term of the equation (17) means, from left to right, fuel consumption by combustion reaction, accumulation of fuel mass, fuel mass inflowing from the upstream, fuel mass outflowing to the downstream, and fuel mass increase by mixture gas flow rate increase, respectively. Here, assume complete combustion, namely, the second terms of the right hand sides of the equations (17) and (18) are to be considered as zero. For an assumption consistency, all of fuel is assumed to react completely at this region. Hence, by taking account of the quasi-static relations $q^{\prime}=(\bar{q} / \bar{w}) w^{\prime}$ and $w^{\prime}=N(\bar{w} / \bar{Y}) Y^{\prime}$, the second term of the left hand side of the equation (17) can be approximated as $\bar{\rho} \hat{Y}^{\prime} \cong \bar{\rho} \bar{Y} w^{\prime} / N \bar{w}$. Further, assume an enough thin combustion region. And then, regard each integrand as its average over the integral interval. The equation (17) can be integrated as follows:

$$
\hat{q}^{\prime}+i \omega \sigma \hat{q}^{\prime}=\frac{\bar{\rho} \bar{v} \bar{Y}}{2 \varepsilon \bar{w}} \bar{q} \lambda \hat{\varphi}^{\prime} \exp (-i \omega \tau)+\bar{q} \frac{\hat{v}^{\prime}}{\bar{v}}, \quad \sigma \equiv \frac{\bar{\rho} \bar{Y}}{N \bar{w}}, \quad \tau \equiv \int_{\xi_{u, N}}^{\xi_{u, F}} \frac{1}{\bar{v}} \mathrm{~d} \xi_{u}
$$

The time parameter $\tau$ means the advection delay time of fuel/air ratio fluctuation which flowing along with the advection $\bar{v}$ until the flame from the fuel nozzle. The time parameter $\sigma$ is related to the reaction delay time against fuel mass fluctuation. According to the integral (18), the coefficient of the first term of the right hand side of the equation (19) can be obtained as $\bar{\rho} \bar{v} \bar{Y} / 2 \varepsilon \bar{w}=1$. Hence, the linear heat release fluctuation can be evaluated as follows, where an approximation $m^{\prime} / \bar{m} \cong u_{N}^{\prime} / \bar{u}_{N}$ has been applied: 


$$
\frac{\hat{q}^{\prime}}{\bar{q}}=N_{C} \frac{\overline{\mathbf{u}} \cdot \hat{\mathbf{u}}^{\prime}}{|\overline{\mathbf{u}}|^{2}}+\lambda N_{C} \hat{\varphi}^{\prime} \exp (-i \omega \tau), N_{C} \equiv \frac{1}{1+i \omega \sigma}, \hat{\varphi}^{\prime} \equiv\left(\frac{\hat{u}_{N}^{\prime}}{\bar{u}_{N}}-\frac{\overline{\mathbf{u}} \cdot \hat{\mathbf{u}}^{\prime}}{|\overline{\mathbf{u}}|^{2}}\right)_{\mathbf{x}=\mathbf{x}_{N}}
$$

\subsection{Boundary conditions}

Boundary conditions are considered to be given as the acoustic impedance $Z$. That is,

$$
\hat{p}^{\prime}=Z \mathbf{u}^{\prime} \cdot \mathbf{n}, \quad \hat{p}^{\prime}=Z_{N} \hat{u}_{N}^{\prime}
$$

where $\mathbf{n}$ and $Z_{N}$ mean the outward normal unit vector and the acoustic impedance of the fuel nozzle, respectively. Generally, impedance is complex number.

\subsection{Summary of basic equations of combustion-acoustic system}

By substituting the equation (20), the acoustic wave equation (8) can be written as

$$
\begin{aligned}
& \nabla^{2} \hat{p}^{\prime}+k^{2} \hat{p}^{\prime}=-i k \frac{\gamma-1}{c} \hat{Q}^{\prime}, \\
& \hat{Q}^{\prime}=\left(\frac{\bar{q}}{|\overline{\mathbf{u}}|} \mathbf{G}_{F}+\frac{\bar{q}}{|\overline{\mathbf{u}}|_{\mathbf{x}=\mathbf{x}_{N}}} \mathbf{G}_{N}\right) \cdot \hat{\mathbf{u}}^{\prime}+\frac{\bar{q}}{|\overline{\mathbf{u}}|_{\mathbf{x}=\mathbf{x}_{N}}} H_{N} \frac{\hat{p}^{\prime}}{\bar{\rho} c},
\end{aligned}
$$

where assume a short distance between the nozzle and the flame than the sound wave length $\omega\left(\xi_{u, F}-\xi_{u, N}\right) / c \cong 0$, and thus, the approximations $\hat{p}^{\prime}\left(\mathbf{x}_{N}\right) \cong \hat{p}^{\prime}$ and $\hat{\mathbf{u}}^{\prime}\left(\mathbf{x}_{N}\right) \cong \hat{\mathbf{u}}^{\prime}$ have been considered. The normalized heat release transfer functions including at the equation (23) are

$$
\begin{aligned}
& \mathbf{G}_{F} \equiv-\frac{i \omega \sigma}{1+i \omega \sigma} \frac{\overline{\mathbf{u}}}{|\overline{\mathbf{u}}|}, \quad \mathbf{G}_{N} \equiv-\left.\frac{\lambda \exp (-i \omega \tau)}{1+i \omega \sigma} \frac{\overline{\mathbf{u}}}{|\overline{\mathbf{u}}|}\right|_{\mathbf{x}=\mathbf{x}_{N}}, \\
& \left.H_{N} \equiv \frac{\lambda \exp (-i \omega \tau)}{1+i \omega \sigma} \frac{\bar{\rho} c}{Z_{N}} \frac{|\overline{\mathbf{u}}|}{\bar{u}_{N}}\right|_{\mathbf{x}=\mathbf{x}_{N}},
\end{aligned}
$$

where the nozzle impedance (21) has been considered. By substituting the linearized equation of motion (3), the basic equations can be obtained as follows:

$$
\begin{array}{ll}
c^{2} \nabla^{2} \hat{p}^{\prime}+\omega^{2} \hat{p}^{\prime}=-\alpha \cdot \nabla \hat{p}^{\prime}-i \omega \beta \hat{p}^{\prime}, & \hat{p}^{\prime}=\frac{i Z}{\bar{\rho} \omega} \nabla \hat{p}^{\prime} \cdot \mathbf{n}, \\
\alpha \equiv-\frac{(\gamma-1) \bar{q}}{\bar{\rho}|\overline{\mathbf{u}}|} \mathbf{G}_{F}-\frac{(\gamma-1) \bar{q}}{\bar{\rho}|\overline{\mathbf{u}}|_{\mathbf{x}=\mathbf{x}_{N}}} \mathbf{G}_{N}, \quad \beta \equiv \frac{(\gamma-1) \bar{q}}{|\overline{\mathbf{u}}|_{\mathbf{x}=\mathbf{x}_{N}}} \frac{H_{N}}{\bar{\rho} c} .
\end{array}
$$

When reduced the spatial dimension, this three-dimensional equation is consistent with the one-dimensional equation of the prior paper [3].

If assuming $\hat{p}^{\prime}\left(\mathbf{x}_{N}\right) \cong \hat{p}^{\prime}, \quad \hat{\mathbf{u}}^{\prime}\left(\mathbf{x}_{N}\right) \cong \hat{\mathbf{u}}^{\prime}$, and $\left|\overline{\mathbf{u}}\left(\mathbf{x}_{N}\right)\right| \cong|\overline{\mathbf{u}}|$ near combustion region, and, high nozzle impedance $\hat{u}_{N}^{\prime} \cong 0$, the heat release fluctuation (20) can be approximated as the similar form of $N-\tau$ model of Culick [8]. The discussions in this section can be said concerning to the mechanism of coefficient $N_{C}$ of the equation (20). In the conventional $N-\tau$ model, the coefficient $N_{C}$ is corresponding to the reaction index and has been defined as the real number. This paper has corrected this model with consideration of the reaction delay time as above. 


\section{Formulations of finite element models of combustion-acoustic system}

For precise analysis, we have to consider the three-dimensional geometry of combustor and parameters. To achieve this, finite element method can be applied. In this section, reformulations of basic equations (26) as finite element models are discussed.

\subsection{Weakly form of the integral equation of combustion-acoustic system}

Consider the approximate solution $\hat{p}^{\prime}$ of the equation (26). By multiplied a weighting function $W$ and then integrating the equation, a residue $\delta$ appears, because the approximate solution is considered and the equation is not satisfied strictly. The rank of differential can be reduced by Gauss integral formula, and further, the boundary condition can be substituted, and hence the weakly form of the integral equation can be written as follows, where $\mathbf{x}, \Omega$ and $\Gamma$ are three-dimensional spatial coordinates, volumetric range to be evaluated, and the boundary surface of the volumetric range $\Omega$, respectively:

$$
\begin{aligned}
& \omega^{2} \int_{\Omega} W \hat{p}^{\prime} \mathrm{d} \mathbf{x}-\int_{\Omega} \nabla\left(c^{2} W\right) \cdot \nabla \hat{p}^{\prime} \mathrm{d} \mathbf{x} \\
& -i \omega \int_{\Gamma} \frac{\bar{\rho} c}{Z} W c \hat{p}^{\prime} \mathrm{d} \mathbf{x}+\int_{\Omega} W \alpha \cdot \nabla \hat{p}^{\prime} \mathrm{d} \mathbf{x}+i \omega \int_{\Omega} W \beta \hat{p}^{\prime} \mathrm{d} \mathbf{x}=\delta .
\end{aligned}
$$

\subsection{Finite element model derived from discretization of the integral equation}

Consider variables which are defined discretely on the nodes of computational grid, and interpolated between the nodes by the polynomial. The integral over the volumetric range $\Omega$ of the equation (29) can be evaluated as the superposition of the integrals of each small elements divided by the computational grid. That is,

$$
\begin{aligned}
& \hat{p}^{\prime}(\mathbf{x})=\sum_{m=1}^{M} N_{m}(\mathbf{x}) p_{m}, \quad W(\mathbf{x})=\sum_{m=1}^{M} N_{m}(\mathbf{x}) W_{m}, \\
& \int_{\Omega} \mathrm{d} \mathbf{x}=\sum_{l} \int_{\Omega_{l}} \mathrm{~d} \mathbf{x}, \quad \int_{\Gamma} \mathrm{d} \mathbf{x}=\sum_{l} \int_{\Gamma_{l}} \mathrm{~d} \mathbf{x},
\end{aligned}
$$

where $p_{m}$ and $N_{m}(\mathbf{x})$ mean the variable defined discretely on the nodes and the polynomial interpolating between the nodes, respectively. Hereafter, $l$ means the element number of finite element model. The integral equation (29) is discretized by the equation (30). And then by choosing the weights $w_{m}$ which minimize the residue $\partial \delta / \partial W_{m}=0$, the finite element model can be obtained as

$$
\begin{aligned}
& \omega^{2}\left\{M_{m n}\right\}\left\{p_{n}\right\}-\left\{K_{m n}\right\}\left\{p_{n}\right\} \\
& -\omega\left\{C_{Z, m n}\right\}\left\{p_{n}\right\}+\left\{C_{G, m n}\right\}\left\{p_{n}\right\}+\omega\left\{C_{H, m n}\right\}\left\{p_{n}\right\}=0,
\end{aligned}
$$

where \{\} means the matrix, and elements of each matrices are respectively

$$
\begin{aligned}
& M_{m n} \equiv \sum_{l} I_{1, m n, l}, \quad K_{m n} \equiv \sum_{l} \widetilde{c}_{l}^{2} I_{2, m n, l}, \\
& C_{Z, m n} \equiv \sum_{l} i \frac{\widetilde{c}_{l}}{\widetilde{Z}_{l}} I_{3, m n, l}, \quad C_{G, m n} \equiv \sum_{l} \widetilde{\alpha}_{l} \cdot \mathbf{I}_{4 m n, l}, C_{H, m n} \equiv \sum_{l} i \widetilde{\beta}_{l} I_{1, m n, l}, \\
& \widetilde{c}_{l} \equiv \frac{1}{\Omega_{l}} \int_{\Omega_{l}} c \mathrm{~d} \mathbf{x}, \\
& \frac{1}{\widetilde{Z}_{l}} \equiv \frac{1}{\Gamma_{l}} \int_{\Gamma_{l}} \frac{\bar{\rho} c}{Z} \mathrm{~d} \mathbf{x}, \quad \widetilde{\alpha}_{l} \equiv \frac{1}{\Omega_{l}} \int_{\Omega_{l}} \alpha \mathrm{d} \mathbf{x}, \quad \widetilde{\beta}_{l} \equiv \frac{1}{\Omega_{l}} \int_{\Omega_{l}} \beta \mathrm{d} \mathbf{x},
\end{aligned}
$$




$$
\begin{array}{rlrl}
I_{1, m n, l} & \equiv \int_{\Omega_{l}} N_{m} N_{n} \mathrm{~d} \mathbf{x}, & I_{2, m n, l} \equiv \int_{\Omega_{l}} \nabla N_{m} \cdot \nabla N_{n} \mathrm{~d} \mathbf{x}, \\
I_{3, m n, l} \equiv \int_{\Gamma_{l}} N_{m} N_{n} \mathrm{~d} \mathbf{x}, & \mathbf{I}_{4, m n, l} \equiv \int_{\Omega_{l}} N_{m} \cdot \nabla N_{n} \mathrm{~d} \mathbf{x},
\end{array}
$$

where the following approximation with respect to the integration of the product of the variables has been considered:

$$
\int_{\Omega_{l}} F G \mathrm{~d} \mathbf{x} \cong \frac{1}{\Omega_{l}} \int_{\Omega_{l}} F \mathrm{~d} \mathbf{x} \int_{\Omega_{l}} G \mathrm{~d} \mathbf{x}, \quad \int_{\Gamma_{l}} F G \mathrm{~d} \mathbf{x} \cong \frac{1}{\Gamma_{l}} \int_{\Gamma_{l}} F \mathrm{~d} \mathbf{x} \int_{\Gamma_{l}} G \mathrm{~d} \mathbf{x}
$$

The parameters $\widetilde{c}_{l}, \widetilde{Z}_{l}, \widetilde{\alpha}_{l}$, and $\widetilde{\beta}_{l}$ of the equations (35) and (36) are assumed those are evaluated by the heat release transfer functions (27) with results of computational fluid dynamics simulation and then those are averaged at element by element of finite element mesh model. Only elements that flame is present at that position, non-zero parameters of $\widetilde{\alpha}_{l}$ and $\widetilde{\beta}_{l}$ are given. This finite element analysis can consider the followings:

1. Acoustic behavior as the basis of model;

2. Three-dimensional precise structure of a combustor and distribution of sound speed;

3. Acoustic characteristic at the boundary of combustor, e.g. the combustor end and the combustor wall;

4. Three-dimensional distribution of heat release fluctuation model at element by element of finite element model.

\subsection{Numerical features of finite element model of combustion-acoustic system}

Finite element model (32) consists of complex matrices. Scale of each matrix is the same as $M \times M$ depending on the number of nodes $M$ of mesh model. In practice problems of an actual gas turbine combustor, the number of nodes tends to be large to simulate the precise combustor geometry, generally about 10 mega nodes. Hence, scale of each matrices of finite element model (32) is assumed as more than $10^{7} \times 10^{7}$ in practice problems.

The finite element model (32) has eigenvalues, which are complex numbers as mentioned below. The real part and imaginary part of eigenvalues are corresponding to the resonance frequencies and the damping rate of the system, respectively. The instability of the system can be evaluated by solving the eigenvalue problem of the finite element model. The solution method of eigenvalue problem depends on the form and features of matrix.

The mass matrix $\left\{M_{m n}\right\}$ and the elasticity matrix $\left\{K_{m n}\right\}$ are respectively real symmetric matrix, so that the matrix $I_{1, m n, l}=I_{1, n m, l}, I_{2, m n, l}=I_{2, n m, l}$ and $\widetilde{c}_{l}$ are real and symmetric. The impedance matrix $\left\{C_{Z, m n}\right\},\left\{C_{G, m n}\right\}$ and $\left\{C_{H, m n}\right\}$ are complex and non-Hermitian, so that the parameters $\alpha, \beta$ and $Z$ are complex and the matrix $I_{1, m n, l} \neq I_{1, n m, l}^{*}$ and $I_{2, m n, l} \neq I_{2, n m, l}^{*}$ are not Hermitian, where superscript of asterisk means complex conjugate. We should solve the complex eigenvalue problem of non-Hermitian matrix. However, complex eigenvalue analysis of large scale non-Hermitian matrix is not easy in terms of calculation cost and accuracy.

\section{Instability analysis method}

In this section, the solution of the calculation cost and accuracy problem of large scale complex eigenvalue analysis is discussed. 


\subsection{Complex eigenvalue analysis method}

At first, solve the real eigenvalue problem of finite element model without considering the impedance matrices of the causes of complex eigenvalues. At such problems, the equation (32) can be written as

$$
\omega^{2}\left\{M_{m n}\right\}\left\{p_{n}\right\}-\left\{K_{m n}\right\}\left\{p_{n}\right\}=0 \quad(m=1,2, \cdots, M \text { and } n=1,2, \cdots, M)
$$

and real eigenvectors $\left\{p_{n}\right\}_{m}(m=1,2, \cdots, M)$ can be obtained. Eigenvectors of a real symmetric matrix problem have orthogonality as $\left\{p_{n}\right\}_{a} \cdot\left\{p_{n}\right\}_{b}=0(a \neq b)$. Arbitrary vectors which are not orthogonal to all real eigenvectors $\left\{p_{n}\right\}_{m}$ can be represented as the superposition of this orthogonal vector series. The complex eigenvector of finite element model (32) should be also able to be represented as the superposition of real eigenvectors. Furthermore, as long as it is not very higher mode, complex eigenvectors should be also able to be represented accurately by only lower order real eigenvectors. By neglected higher real eigenvectors, scale of problem can be reduced. Hereafter, these real eigenvectors and the corresponding real eigenvalues will be referred to as "base vectors" and "base eigenvalue", respectively. So, consider the solution of the complex eigenvalue problem (32) as the superposition of base vectors. That is,

$$
\left\{p_{n}\right\}=\left\{p_{n m}\right\}\left\{\kappa_{m}\right\} \quad(m=1,2, \cdots, \mu \text { and } n=1,2, \cdots, M)
$$

where $\left\{p_{n m}\right\}$ is the non-square matrix which consists of column matrices of base vectors, namely $\left\{p_{n m}\right\}=\left\{\begin{array}{llll}\left\{p_{n}\right\}_{1} & \left\{p_{n}\right\}_{2} & \cdots & \left\{p_{n}\right\}_{\mu}\end{array}\right\}$. The superposition (41) is an approximation which has been neglected higher modes $\mu(<M)$. The matrix $\left\{\kappa_{m}\right\}$ is the weight to represent the complex eigenvector by the superposition of base vectors. Solving this weight, the solution of complex eigenvector can be determined as the equation (41).

Substituting the approximation (41) into the complex eigenvalue problem (32) and multiplying the transposition matrix $\left\{p_{n m}\right\}^{T}$, the following equation can be obtained:

$$
\begin{aligned}
& \omega^{2}\left\{M_{m n}^{\prime}\right\}\left\{\kappa_{m}\right\}-\left\{K_{m n}^{\prime}\right\}\left\{\kappa_{m}\right\} \\
& -\omega\left\{C_{Z, m n}^{\prime}\right\}\left\{\kappa_{m}\right\}+\left\{C_{G, m n}^{\prime}\right\}\left\{\kappa_{m}\right\}+\omega\left\{C_{H, m n}^{\prime}\right\}\left\{\kappa_{m}\right\}=0 \quad(m, n=1,2, \cdots \mu), \\
& \left\{M_{m n}^{\prime}\right\} \equiv\left\{p_{n m}\right\}^{T}\left\{M_{m n}\right\}\left\{p_{n m}\right\}, \quad\left\{K_{m n}^{\prime}\right\} \equiv\left\{p_{n m}\right\}^{T}\left\{K_{m n}\right\}\left\{p_{n m}\right\}, \\
& \left\{C_{X, m n}^{\prime}\right\} \equiv\left\{p_{n m}\right\}^{T}\left\{C_{X, m n}\right\}\left\{p_{n m}\right\} \quad(X=Z, G, H) .
\end{aligned}
$$

The matrix size can be reduced from $M \times M$ to $\mu \times \mu$ as of the equation (42). By choosing appropriate real eigenvectors necessary for representing the complex eigenvectors precisely, the accuracy of analysis would be able not to be worsened. Finally, to calculate complex eigenvalues, reformulate the eigenvalue problem (42) to the general form as

$$
\begin{aligned}
& \omega\left\{A_{m n}\right\}\left\{\theta_{m}\right\}=\left\{B_{m n}\right\}\left\{\theta_{m}\right\}, \\
& \left\{A_{m n}\right\} \equiv\left\{\begin{array}{cc}
\left\{C_{H, m n}^{\prime}\right\}-\left\{C_{Z, m n}^{\prime}\right\} & \left\{M_{m n}^{\prime}\right\} \\
\left\{M_{m n}^{\prime}\right\} & 0
\end{array}\right\}, \\
& \left\{B_{m n}\right\} \equiv\left\{\begin{array}{cc}
\left\{K_{m n}^{\prime}\right\}+\left\{C_{G, m n}^{\prime}\right\} & 0 \\
0 & \left\{M_{m n}^{\prime}\right\}
\end{array}\right\}, \quad\left\{\theta_{m}\right\} \equiv\left\{\begin{array}{c}
\left\{\kappa_{m}\right\} \\
\omega\left\{\kappa_{m}\right\}
\end{array}\right\} .
\end{aligned}
$$

Elements of the impedance matrix $\left\{C_{Z, m n}^{\prime}\right\},\left\{C_{G, m n}^{\prime}\right\}$ and $\left\{C_{H, m n}^{\prime}\right\}$ are variables with respect to frequency. By repeating the eigenvalue calculation with assuming a frequency of the impedance matrices until the solution will be converged, the solution can be obtained. 


\section{Validation at a simple tube}

In this section, the validation of the method introduced at Section 4 to evaluate complex eigenvalues of a large non-Hermitian matrix approximately is discussed.

\subsection{Validation model}

Consider a non-combustion tube of length $L$ with a closed and an finite impedance ends. Such a case, in particular about longitudinal modes, the acoustic equation (22) can be solved analytically [3], and then, the eigenvalues $f_{m}=\omega_{m} / 2 \pi$, the resonance frequencies $f_{R, m}$, the damping ratios $\zeta_{m}$ and the acoustic mode shape $\hat{p}_{m}^{\prime}$ can be obtained as

$$
\begin{aligned}
& f_{m}=\frac{c}{4 \pi L} \arg (R)-i \frac{c}{4 \pi L} \ln |R|, \quad R=\frac{Z / \bar{\rho} c-1}{Z / \bar{\rho} c+1}, \\
& f_{R, m}=\frac{c}{4 \pi L} \arg (R), \quad \zeta_{m}=-\frac{c}{4 \pi L f_{R, m}} \ln |R|, \quad \hat{p}_{m}^{\prime}=\cos \left(\omega_{m} x / c\right),
\end{aligned}
$$

where $Z$ and $R$ are the acoustic impedance and the acoustic reflection coefficient of the end, respectively. By given a finite impedance $Z$, eigenvalues would be complex numbers.

Two types of mesh models as shown in Figure 3 are considered. Each mesh models are axial uniform. The fine mesh model is divided axially by 19 cross-sections, and has 2,057 nodes. The coarse mesh model is divided by 9 cross-sections, and has 803 nodes.

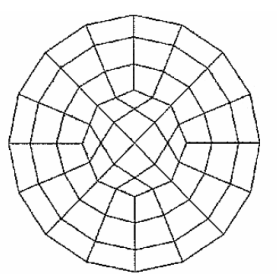

a) Coarse mesh model

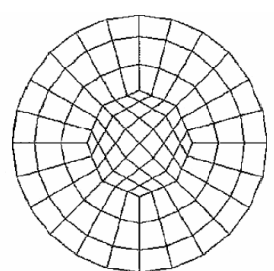

b) Fine mesh model

Fig. 3 Cross-sectional schematic of finite element mesh model of a tube.

\subsection{Accuracy of real eigenvalues evaluation}

To evaluate accurate complex eigenvalues, base vectors should be evaluated accurately. So, the analysis accuracy of base vectors should be make sure, at first. Table 1 shows the base eigenvalues evaluated by finite element model in case that the tube length $L$, the tube radius $a$, sound speed $c$, and the boundary conditions at both ends are assumed as $1 \mathrm{~m}$, $0.35 \mathrm{~m}, 340 \mathrm{~m} / \mathrm{s}$, and rigid ends, respectively. Theoretical results in Table 1 were evaluated as

$$
f_{l, m}=\frac{c}{2 \pi} \sqrt{\left(\frac{l \pi}{L}\right)^{2}+\beta_{m}{ }^{2}},
$$

where $l$ and $m$ are a longitudinal mode number and a circumferential mode number. The parameter $\beta_{m}$ is satisfied $\partial J_{m}\left(\beta_{m} r\right) / \partial r=0$ at $r=a$, where $J_{m}($.$) is Bessel function.$

Figure 4 shows analysis errors which is defined as the difference of numerical and theoretical results normalized by theoretical results shown in Table 1, and where " $(l, m)$ mode" means the $l$ th longitudinal and $m$ th circumferential mode. The wave length of longitudinal mode and circumferential mode are evaluated as the sound speed divided by the resonance frequency $c / f_{l, 0}$ and the circumference length $2 \pi a$, respectively. As shown in Figure 4, if a finer mesh, more accurate results can be expected. 
Table 1 Real eigenvalue of a tube with rigid ends.

\begin{tabular}{|c|c|c|c|c|}
\hline \multicolumn{2}{|c|}{ Mode number } & \multirow{2}{*}{$\begin{array}{c}\text { Theoretical } \\
\text { results }\end{array}$} & \multicolumn{2}{|c|}{ Finite element analysis results } \\
\hline Longitudinal $l$ & Circumferential $m$ & & 2,057 nodes & 803 nodes \\
\hline 0 & 0 & $0.0 \mathrm{~Hz}$ & $0.079 \mathrm{~Hz}$ & $0.102 \mathrm{~Hz}$ \\
\hline 1 & 0 & $170.0 \mathrm{~Hz}$ & $170.2 \mathrm{~Hz}$ & $170.7 \mathrm{~Hz}$ \\
\hline 2 & 0 & $340.0 \mathrm{~Hz}$ & $341.7 \mathrm{~Hz}$ & $345.6 \mathrm{~Hz}$ \\
\hline 3 & 0 & $510.0 \mathrm{~Hz}$ & $515.9 \mathrm{~Hz}$ & $529.0 \mathrm{~Hz}$ \\
\hline 0 & 1 & $569.0 \mathrm{~Hz}$ & $576.0 \mathrm{~Hz}$ & $582.0 \mathrm{~Hz}$ \\
\hline 1 & 1 & $593.8 \mathrm{~Hz}$ & $601.6 \mathrm{~Hz}$ & $606.0 \mathrm{~Hz}$ \\
\hline 2 & 1 & $662.8 \mathrm{~Hz}$ & $670.8 \mathrm{~Hz}$ & $677.0 \mathrm{~Hz}$ \\
\hline
\end{tabular}

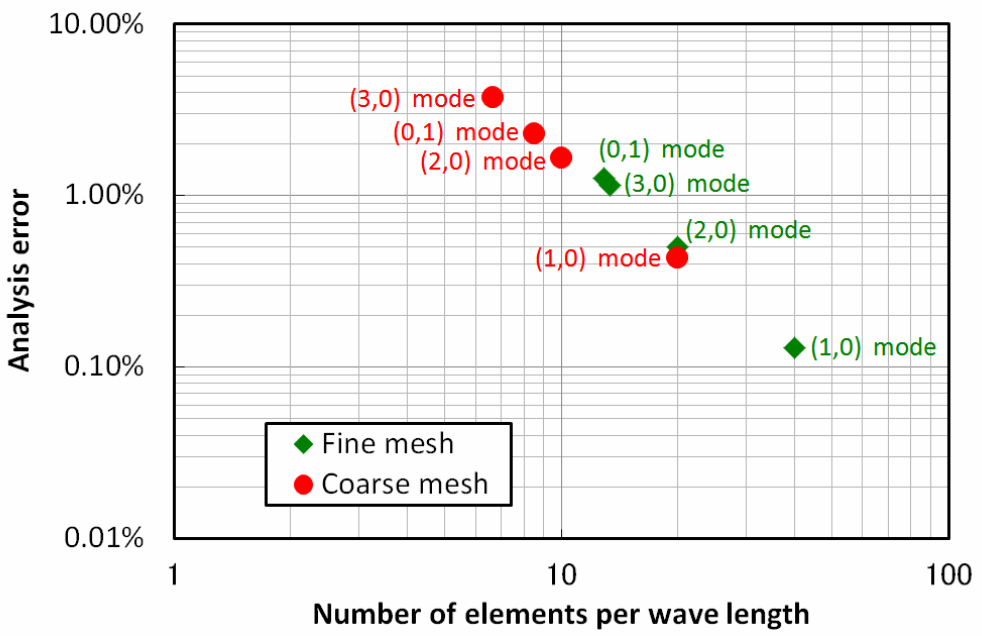

Fig. 4 Analysis error of the real eigenvalues shown in Table 1.

\subsection{Accuracy of complex eigenvalues evaluation}

Figure 5 shows the complex eigenvalues evaluated by finite element analysis (42) approximated with the superposition of base vectors of the coarse mesh model above. Figure 6 shows the complex acoustic mode shapes, which are normalized by the maximum value of each mode shapes. Calculation conditions of these results are shown in Table 2.

In Figure 5 and 6, only the longitudinal modes were chosen as base vectors. Because $0^{\text {th }}$ mode having spatial uniform distribution was also considered, the number of base vectors was $\mu+1$. The numerical results of Figure 5 and 6 were agreed with the theoretical results well despite an approximation by at most six eigenvectors. However, when the number of base vectors was smaller as shown in the case of $\mu=1$ in Figure 6, the analysis accuracy extremely decreased. To ensure accuracy, it seems necessary to consider higher order modes which are sufficient to represent the complex eigenvector. Theoretically, when the complex eigenvector is nearly orthogonal to all of the real eigenvectors unfortunately, for example, when the acoustic impedance of both tube ends are near zero, the approximation can't ensure accuracy. However, in the actual gas turbine combustor which has the mean flow, such situation is rarely, and hence, the concern above would not become an actual problem. Summarizing the results of validations, the following remarks should be care:

1. At first, the real eigenvector should be solved accurately without considering the combustion model and finite impedance at the boundary. To ensure accuracy of this calculation, mesh size would be finer. Therefore, large matrices calculation is needed. Because the matrices are real symmetric, if very fine and large mesh model is applied, the computational efficiency would not significantly affect; 
2. Large scale matrices are not needed to ensure accuracy of the complex eigenvalues, even if those have been needed to ensure accuracy of the real eigenvectors. Matrix scale of the complex eigenvalue calculation can reduced from $M \times M$ depending on the number of mesh nodes $M$ to $\mu \times \mu$ depending on the number of real eigenvectors $\mu$ to be considered in the approximation proposed in this paper. At the sample case of this section, the matrix scale $803 \times 803$ was able to be reduced to $6 \times 6$ for complex eigenvalue calculation without noticeable accuracy degrade.

Table 2 Calculation conditions of Figure 6 and 7.

Mesh model

Length of system $L$

Sound speed $c$

Acoustic impedance at the end $Z$

Maximum number of the real eigenvectors $\mu$
Coarse mesh of Figure 4

$1 \mathrm{~m}$

$340 \mathrm{~m} / \mathrm{s}$

$(1-i) \rho c$

5 or 1

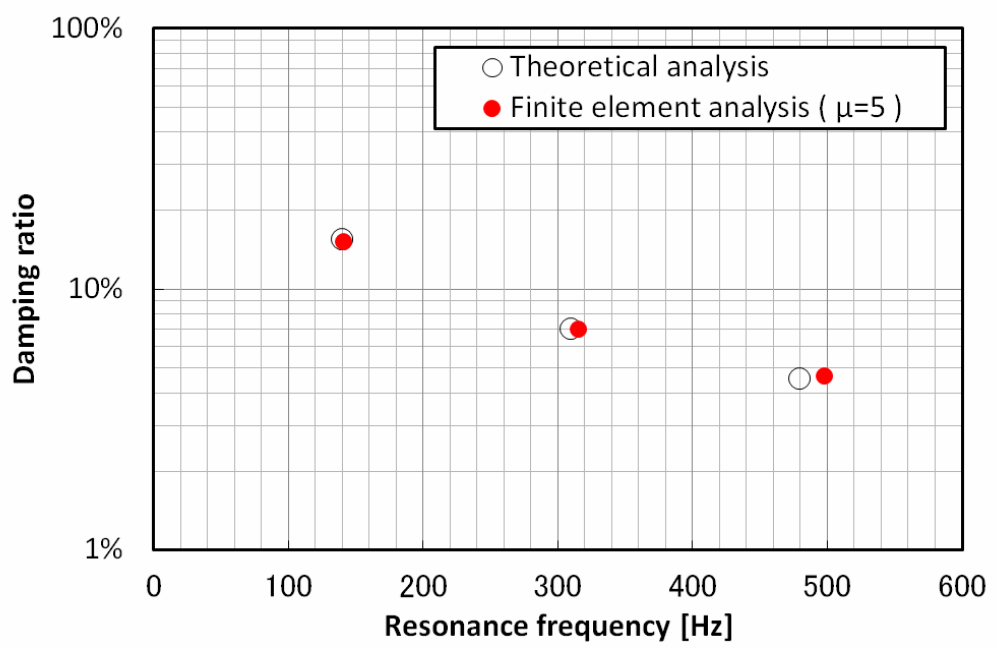

Fig. 5 The complex eigenvalues evaluated by approximated finite element analysis.

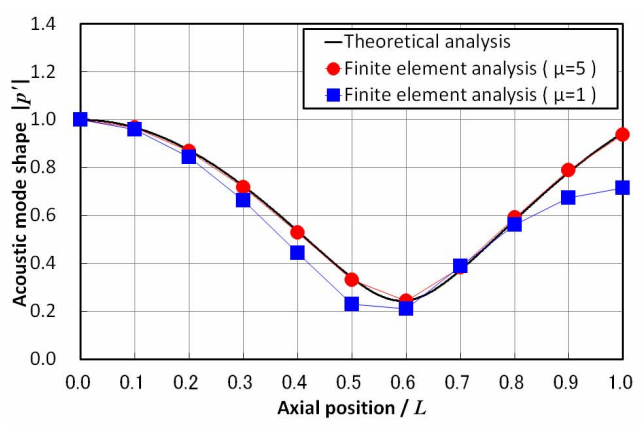

a) $1^{\text {st }}$ mode

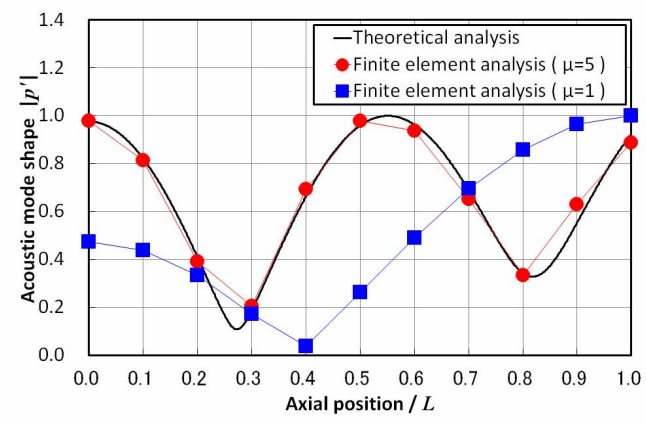

b) $2^{\text {nd }}$ mode

Fig. 6 The complex acoustic mode shapes evaluated by approximated finite element analysis.

\section{Validation at a simple combustor}

In this section, we show the validation results of this method at a simple combustor examples whose theoretical analysis results have been already once evaluated at the prior report [3]. 


\subsection{Validation model}

Consider a cylindrical combustor model having finite impedance ends and the axial coordinates $x$ whose the origin is positioned at the upstream end as shown in Figure 7a. Assume combustion region of width $2 \varepsilon$ at $x=x_{F}$, and, temperature as uniformly $T_{L}$ at the upstream region and $T_{H}$ at the downstream region, respectively. Here, variables with subscript $L$ and $H$ mean those are belong to low temperature $T_{L}$ region and high temperature $T_{H}$ region, respectively. Fuel is assumed to be supplied at $x=x_{N}$, and flows to combustion region while mixing with air. Assume the length and the radius of combustor as $1 \mathrm{~m}$ and $0.045 \mathrm{~m}$, respectively.

The mesh model is shown in Figure $7 \mathrm{~b}$ having 1,717 nodes, which is divided 99 cross-sections. The downstream end is assumed to be opened to the atmosphere and the upstream end of combustor is assumed to be connected to a plenum chamber. The acoustic impedance $Z \equiv \hat{p}^{\prime} / \hat{u}^{\prime}$ at each ends are assumed as

$$
\begin{aligned}
& Z_{3}=Z_{D, R}+\bar{\rho}_{H} c_{H}\left[1-\frac{J_{1}\left(2 k_{H} a\right)}{k_{H} a}+i \frac{H_{1}\left(2 k_{H} a\right)}{k_{H} a}\right], \\
& Z_{0}=Z_{U, R}-i \bar{\rho} c\left(A / A_{C}\right) \cot k L_{C}
\end{aligned}
$$

where $J_{1}(),. H_{1}(), a, A,. A_{C}, L_{C}, Z_{D, R}$ and $Z_{U, R}$ are Bessel function, Struve function, radius of open end, cross-sectional area of combustor, cross-sectional area of chamber, length of chamber, and correlation parameters of the boundary conditions, respectively.

The analysis conditions are shown in Table 3. The heat release model was given uniformly in the cross-section of the one mesh range in the axial direction.

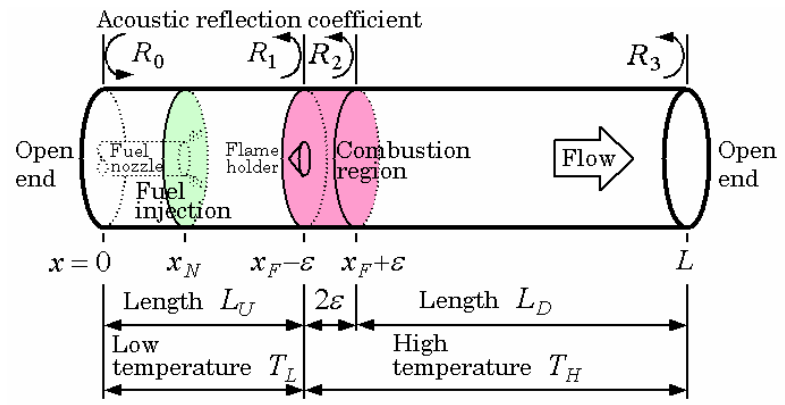

a) Schematic of analysis model

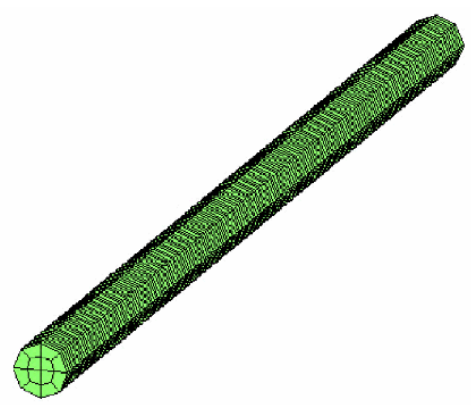

b) Finite element mesh model

Fig. 7 Analysis models.

Table 3 Combustion conditions and analysis parameters

\begin{tabular}{ll}
\hline Fuel type and fuel calorific value & Methane of $40.0 \mathrm{MJ} / \mathrm{m}^{3}$ \\
Fuel flow rate & $4.17 \times 10^{-5} \mathrm{~m}^{3} / \mathrm{sec}$ \\
Heat release density & $q_{0}=1.05 \times 10^{6} \mathrm{~W} / \mathrm{m}^{2}$ \\
Speed of sound & $c_{L}=341 \mathrm{~m} / \mathrm{sec}, c_{H}=440 \mathrm{~m} / \mathrm{sec}$ \\
Gas velocity & $u_{L}=4.50 \mathrm{~m} / \mathrm{sec}, \quad u_{H}=7.52 \mathrm{~m} / \mathrm{sec}$ \\
Reaction delay time & $\sigma=1.11 \times 10^{-1} \mathrm{msec}$ \\
Diffusion factor & $\lambda=0.15$ \\
Additional acoustic resistance of end & $Z_{U, R} / \bar{\rho} c=5.0 \times 10^{-3}, Z_{D, R} / \bar{\rho} c=5.1 \times 10^{-2}$ \\
Nozzle position & $x_{N}=0 \mathrm{~m}$ \\
\hline
\end{tabular}




\subsection{Theoretical analysis}

Assume the spatial distribution of the mean heat release density $\bar{q}$ as

$$
\begin{aligned}
& \bar{q}=q_{0} \delta_{*}\left(x-x_{F}, 2 \varepsilon\right), \quad q_{0} \equiv \frac{1}{A} \int_{V} \bar{q} \mathrm{~d} V, \\
& \delta_{*}\left(x-\xi_{1}, \xi_{2}\right) \equiv \frac{1}{\xi_{2}}\left[h\left(x-\xi_{1}+\xi_{2} / 2\right)-h\left(x-\xi_{1}-\xi_{2} / 2\right)\right],
\end{aligned}
$$

where $h(\cdot)$ means Heaviside's step function. One-dimensional acoustic wave equation is derived by dimension reduction from three-dimensional equation (22) as

$$
\begin{aligned}
& \frac{\partial^{2} \hat{p}^{\prime}}{\partial x^{2}}+k^{2} \hat{p}^{\prime}=-\eta \frac{\partial \hat{p}^{\prime}}{\partial x} \delta_{*}\left(x-x_{F}, 2 \varepsilon\right), \\
& \eta \equiv-\frac{\gamma-1}{\bar{\rho} \bar{u} c^{2}} q_{0} G_{N}, G_{N}=\frac{-i \omega \sigma}{1+i \omega \sigma}-\lambda \frac{\exp (-i \omega \tau)}{1+i \omega \sigma}, \quad \tau \equiv \frac{x_{F}-x_{N}}{\bar{u}},
\end{aligned}
$$

where the transfer function $H_{N}$ has been neglected by the assumption of high impedance nozzle $1 / Z_{N} \cong 0$. The linear equation (55) can be solved, and as the results, the complex eigen angular frequencies $\omega_{m}$ in case of negligible thin combustion region $\varepsilon \rightarrow 0$ can be obtained as

$$
\omega_{m}=\frac{c_{L}}{2 L_{U}} \arg \left(R_{0} R_{1}\right)-i \frac{c_{L}}{2 L_{U}} \ln \left|R_{0} \| R_{1}\right|
$$

where $R_{0}$ and $R_{1}$ are acoustic reflection coefficients at the upstream end of combustor and the upstream end of combustion region, respectively. The acoustic reflection coefficient at the upstream end of combustor can be estimated by the acoustic impedance given as the boundary conditions. The acoustic reflection coefficient at the downstream end of combustion region can be estimated by sequential integration of the equation (55) from the downstream end of combustor. The acoustic reflection coefficients can be evaluated as

$$
\begin{aligned}
& R_{0}=\frac{Z_{0}-\bar{\rho}_{L} c_{L}}{Z_{0}+\bar{\rho}_{L} c_{L}}, \quad R_{1}=\frac{R_{2}-\tanh \left[\left(\eta-\ln c_{H} / c_{L}\right) / 2\right]}{1-R_{2} \tanh \left[\left(\eta-\ln c_{H} / c_{L}\right) / 2\right]}, \\
& R_{2}=R_{3} \exp \left(-2 i \omega_{m} L_{D} / c_{H}\right), \quad R_{3}=\frac{Z_{3}-\bar{\rho}_{H} c_{H}}{Z_{3}+\bar{\rho}_{H} c_{H}} .
\end{aligned}
$$

The ratio of real part and imaginary part of eigenvalue (57) is corresponding to the damping ratio of the system.

\subsection{Validation results}

The theoretical analysis results at these conditions have been already once evaluated and compared with test results at the prior reports [3] as shown in Figure 8. The theoretical results in Figure 8 are the response magnification defined by the reciprocal of damping ratio. The thin line in Figure 8 is the theoretical results with neglected advection delay time $\tau=0$, which has been normalized by the response magnification at $x_{F}=0.22 \mathrm{~m}$. The bold line, which is considered advection delay time $\tau=\left(x_{F}-x_{N}\right) / u_{L}$, has been also normalized by the same value as the thin line, namely the response magnification of thin line at $x_{F}=0.22 \mathrm{~m}$.

Figure 9 shows the numerical results evaluated by finite element model, which has been normalized by the same value as theoretical results. The numerical results of Figure 9 was 
evaluated while changing the heat release position from $x_{F}=0.1 \mathrm{~m}$ to $x_{F}=0.8 \mathrm{~m}$, where the coordinates $x_{F}$ of finite element model is defined as the position of the upstream end of the mesh given the heat release model. By referenced also this coordinates, the advection delay time $\tau=\left(x_{F}-x_{N}\right) / u_{L}$ was also evaluated and given to the heat release model mesh. The number of base vectors to approximate the complex eigenvalue problem was 100 . The highest longitudinal base vector of finite element model divided by 99 cross-sections is $(50,0,0)$ mode, which is included in 100 lower order modes such an elongated model as shown in Figure $7 \mathrm{~b}$. Namely, all longitudinal base vectors were considered as base vectors to approximate the complex eigenvalue problem. The numerical results by finite element model are found to be almost agreed with the theoretical results as can be seen from Figure 9.

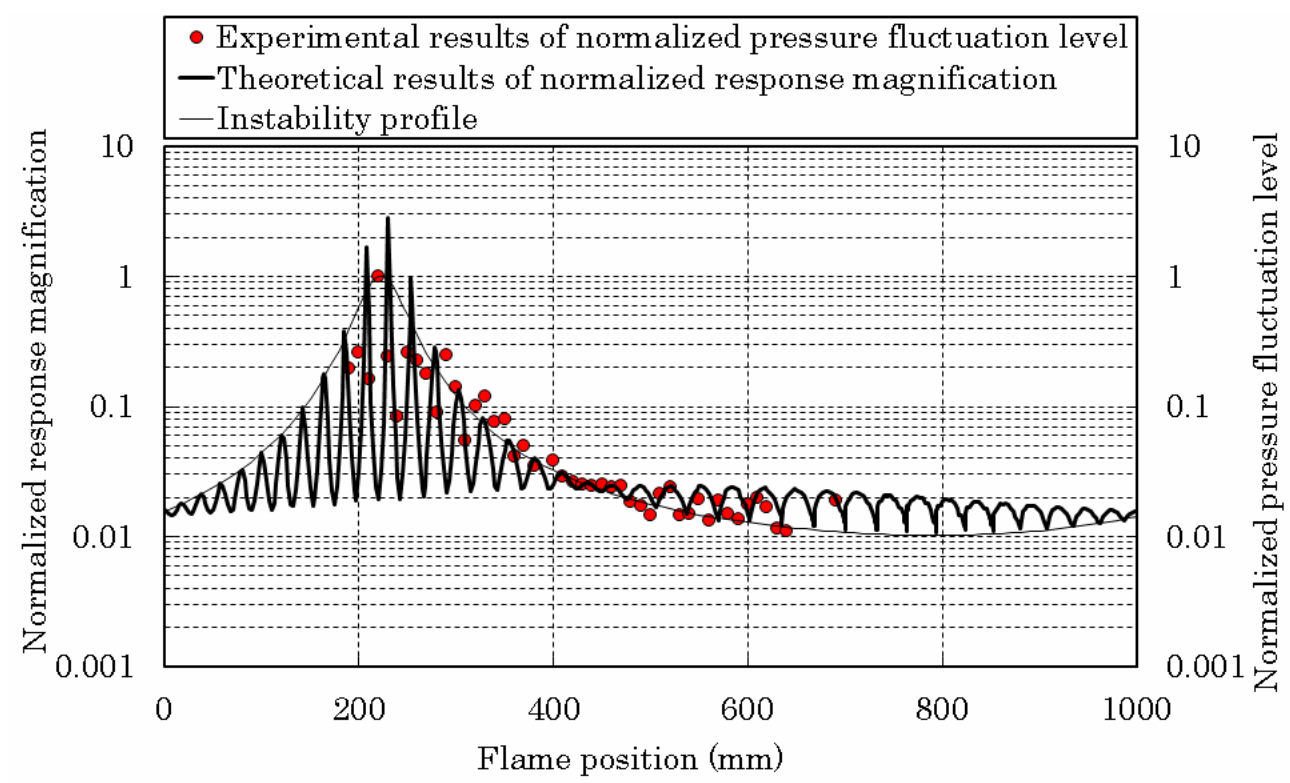

Fig. 8 Theoretical and experimental instability of the 1st. acoustic mode of validation model [3].

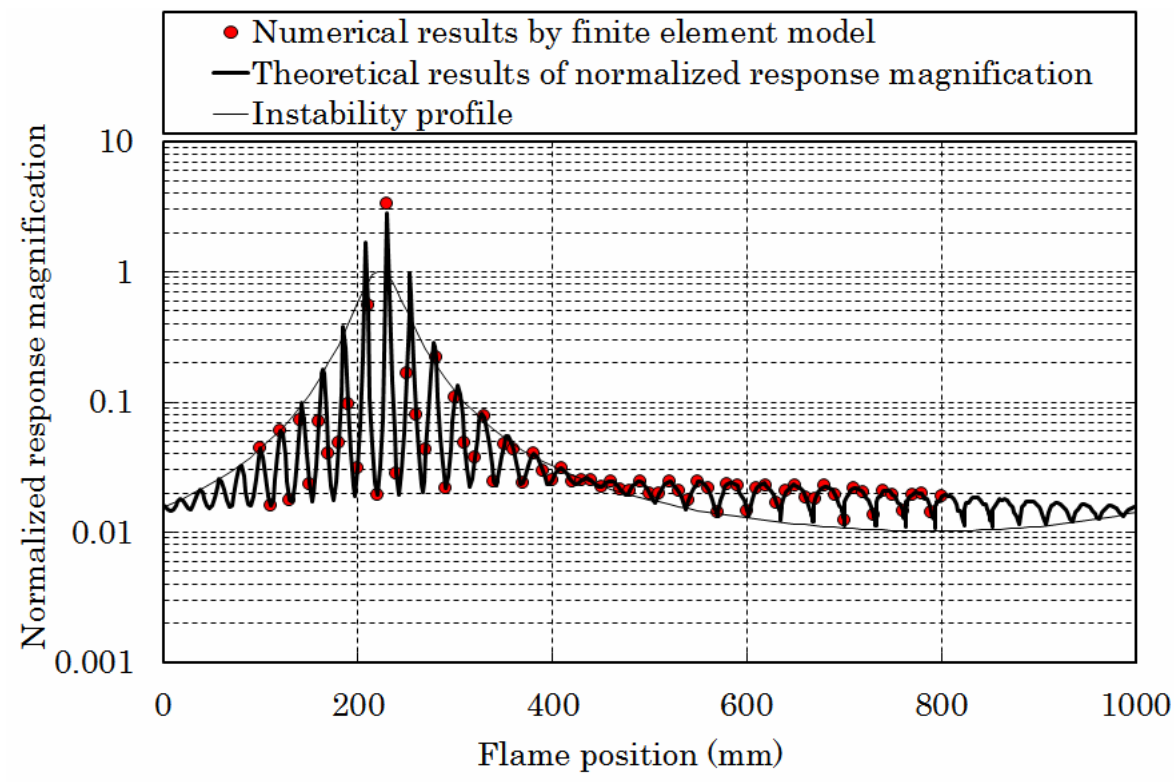

Fig. 9 Comparison of numerical results by finite element model with theoretical results. 


\section{Conclusions}

In this paper, linear instability analysis method with three-dimensional finite element model of combustion-acoustic system is developed by extended from one-dimensional theoretical model which has been discussed at the prior report [3]. This finite element analysis of this paper can consider the followings:

1. Acoustic behavior as the basis of model;

2. Three-dimensional precise structure of a combustor and distribution of sound speed;

3. Acoustic characteristic at the boundary of combustor;

4. Three-dimensional distribution of heat release fluctuation model at element by element of finite element model.

In this paper, with assumed that the mean of combustion has been already obtained by computational fluid dynamics simulation, the perturbation of combustion having a coherency with the acoustic resonance has been formulated. The followings have been assumed. At actual application, whether the assumptions are holding should be always care. Further, verification of these is one of the remaining issues.

1. Longer acoustic wavelength than the scale of advection;

2. Circulation flow or, chemical mechanism itself is not in resonant;

3. Constant diffusion effect of fuel/air ratio fluctuation;

Further, the approximate calculation method for evaluating the complex eigenvalues of finite element model of combustion-acoustic system is proposed and validated at simple examples. The complex eigenvalues of validation examples is shown that be agreed well with the theoretical results, and the accuracy of approximation was verified. Also, the calculation efficiency by approximation was verified. Even if a mesh model were large scale like a whole of all combustors of an actual gas turbine engine, accurate and efficient instability analysis can be expected by the analysis method of this paper.

As can be seen from the analysis results, combustion-acoustic instability is sensitive to slight difference of conditions. Parameters for finite element model of this paper are assumed those are the average of results of computational fluid dynamics simulation at element by element of finite element mesh model. The accuracy improvement of parameter estimation with large scale numerical simulation is one of the remaining issues.

\section{References}

[1] K. Tanaka, K. Nishida and W. Akizuki, "Gas turbine combustor technology contribution to environmental conservation," Mitsubishi Heavy Industries Technical Review, 46(2), 6-12 (2009).

[2] K. Ikeda, K. Matsuyama and M. Nishimura, "Robust Gas Turbine Combustor with Acoustic Liner," J. Thermal Science and Tech., 7(1), 199-210 (2012).

[3] K. Ikeda, K. Matsuyama, M. Isono and M. Nishimura, "Linear Instability Analysis of Lean Premixed Combustion,” J. Thermal Science and Tech., 7(4), 649-664 (2012).

[4] J.W.S. Rayleigh, The theory of sound (Dover, New York, 1945).

[5] A.P. Dowling, "The Calculation of Thermoacoustic oscillations," J. Sound and Vibration, 180(4), 557-581 (1995).

[6] U. Hegde, D. Reuter, and B.T. Zinn, "Sound Generation by Ducted Flames," AIAA journal 26(5), 532-537 (1988).

[7] F.A. Williams, Combustion Theory, 2nd ed. (Benjamin/Cummings, 1985).

[8] F.E.C. Culick and V. Yang, "Overview of combustion instabilities in liquid-propellant rocket engines," Progress in Astronautics and Aeronautics, 169, 3-37 (1995).

[9] S. Akamatsu and A.P. Dowling, "Three dimensional thermoacoustic oscillation in a premix combustor," ASME 2001-GT-0034, Proc. ASME Turbo Expo 2001 (2001).

[10] P.M. Morse and K.U. Ingard, Theoretical Acoustics (McGraw-Hill, New York, 1968). 